hot bodies is a non-coordinated process, and that by far the greatest quantities of energy in the universe are in a non-coordinated form. The lecturer showed that the work of Gibbs in thermodynamics, in particular his generalization of Carnot's law, entitles him to the position of one of the founders of the science of non-coordinated motions.

R. G. D. Richardson, Secretary.

\title{
THE MARCH MEETING OF THE SOCIETY
}

The two hundred thirty-fourth regular meeting of the Society was held at Columbia University on Saturday, March 1, and was preceded, on February 29, by the first Josiah Willard Gibbs Lecture (see this Bulletin, p. 289). The attendance at this meeting included the following forty-seven members of the Society:

Alexander, Babb, Barnum, Bernstein, W. M. Bond, E. W. Brown, R. W. Burgess, Coolidge, Cowley, Eisenhart, Fields, Fite, Gafafer, Gehman, Gilman, Glenn, Grove, Guggenbühl, Hausle, Hill, Hille, Himwich, Huntington, Joffe, Kellogg, Kline, Langman, Harry Levy, Mirick, H. H. Mitchell, C. L. E. Moore, Mullins, Pell, Post, Rainich, Reddick, R. G. D. Richardson, Ritt, Seely, H. D. Thompson, Veblen, H. E. Webb, Wedderburn, Weiss, H. S. White, Whittemore, J. W. Young.

The Secretary announced the election of the following persons to membership in the Society:

Dr. Maria Castellani, Bryn Mawr College;

Miss Laura Guggenbühl, Bryn Mawr College;

Mr. Henry Alfred Hoover, Washington University;

Professor Benjamin Hoffman Kerstein, State Teachers' College Silver City, N. M.;

Professor James Shannon Miller, Emory and Henry College.

Thirty-seven applications for membership were received.

On recommendation of the Council, the following amendment to the By-Laws was presented to the Society: to insert in Section 1 of Article III the words "of ex-presidents, 
for a period of six years after the expiration of their respective presidential terms," so that the section shall read:

"Section 1. The Council shall consist of the officers specified in Article I, of ex-secretaries who have served as Secretary for ten years or more, of ex-presidents for a period of six years after the expiration of their respective presidential terms, and of fifteen additional elected members chosen in accordance with Article IV, Section 2."

The Society voted to adopt this amendment.

At the meeting of the Council, the appointment of the following committees was announced: On arrangements for the Annual Meeting of 1924, to be held at Washington: Professors H. L. Hodgkins (chairman), J. T. Erwin, Archibald Henderson, A. E. Landry, F. D. Murnaghan, and J. M. Page, Miss Elizabeth W. Wilson, and the Secretary; on the Second Award of the Bôcher Prize: Professors G. D. Birkhoff (chairman), L. E. Dickson, and H. S. White.

The report of the Auditing Committee, appointed to examine the accounts of the Treasurer, was presented to the Council for its information; this report shows a balance for the general funds of the Society, as of December 15, 1923 , of $\$ 9,638.78$, of which $\$ 5,726.91$ is held as reserve to protect life memberships and $\$ 2,500$ is estimated as required for printing the outstanding numbers of the BULLETIN and Transactions for 1922 and 1923.

The Committee on the next Colloquium reported a recommendation that it be held at Cornell University in the summer of 1925 ; this recommandation was adopted.

President Veblen presided at the scientific sessions, relieved by Professors E. W. Brown, H. S. White, and VicePresident J. H. M. Wedderburn. Titles and abstracts of the papers read at this meeting follow below. Mr. Van de Walle was introduced be Professor Huntington. Professor Murnaghan's paper was read by Mr. Rainich, and Mr. Van de Walle's by Professor Huntington. The papers of Alexander, Garabedian, Michal, Moore, Murray, Rainich (third paper), and Stone, were read by title. 
1. Professor F. D. Murnaghan: The outer multiplication of integral forms.

The author gives a short proof by means of tensor algebra of a known theorem on the composition of integral forms. In any space $S_{n}$, not necessarily metrical, of $n$ dimensions, the generalised Kronecker symbol $\delta_{s_{1}}^{r_{1} \cdots r_{p}}(p \leqq n)$ may be defined by the following properties. It is alternating in both the upper labels $r_{1} \cdots r_{p}$ and the lower labels $s_{1} \cdots s_{p}$. It has the value zero if the group $r_{1} \cdots r_{p}$ is different from the group $s_{1} \cdots s_{p}$ and has the value \pm 1 according as the arrangement $r_{1} \cdots r_{p}$ is of the same class as the arrangement $s_{1} \cdots s_{p}$ or not. The symbol $\delta_{s_{1} \cdots s_{p}}^{r_{1} \cdots r_{p}}$ defines a mixed tensor of rank $2 p$. If we have two alternating covariant tensors $A_{l_{1} \ldots l_{i}}$ and $B_{m_{1} \ldots m_{j}}$ of ranks $i$ and $j$ respectively $(i+j \leqq n)$ then $\delta_{\alpha_{1} \cdots \alpha_{i} \beta_{1} \cdots \beta_{j}}^{l_{1} \ldots l_{j}} A_{l_{1} \cdots l_{i}} B_{m_{1}} \cdots m_{j}$ is an alternating covariant tensor of rank $i+j$. If we construct integral forms with the components of the alternating covariant tensors as coefficients, the form of rank $i+j$ is the outer product of the two forms of rank $i$ and $j$ respectively. It may be expressly mentioned that the tensor $\delta_{s_{1} \cdots s_{p}}^{r_{1} \cdots r_{p}}$ is non metrical, i.e., the $S_{n}$ need not be Riemannian nor need it have a geometry of paths. Thus the usual presentation, in the special case $p=1$, of $\delta_{s_{1}}^{r_{1}}$ as equal to $g_{s_{1}}^{r_{1}}=g^{r_{1} k} g_{s_{1} k}$ is unfortunate.

2. Mr. G. Y. Rainich: A modification of tensor analysis.

In this paper, vectors are represented by their components with respect to a special system of rectangular axes at each point, and points, as usual by $n$ coordinates. The derivative of a tensor $\varphi$ of rank zero is given by $\left(\partial \varphi / \partial x_{\rho}\right) \cdot b \rho_{i}$, where $b_{i j}=b_{j i}$, and the derivative of a tensor $\psi_{i}$ of the first rank by $\left(\partial \psi_{i} / \partial x \rho\right) \cdot b_{\rho j}+\psi_{\rho} T_{i j}^{\rho}$, etc. If we require that (1) the derivatives of the Galilean tensor $\delta_{j}^{i}$ be zero and (2) the second derivatives of a tensor of rank zero be independent of the order of differentiation, we find that $T_{j k}^{i}$ is one-half the determinant made up of three rows of three terms each: $\partial a_{i \rho} / \partial x \sigma, \cdots ; b_{i \rho}, \cdots ; b_{i} \sigma, \cdots$; where the $\alpha_{i j}$ are the coefficients of the inverse transformation with respect to the $b_{i j}$. There is no distinction between covariant and contravariant quantities. This representa- 
tion and the usual one are connected by the formula $g_{i j}=a_{i \rho} \cdot a_{\rho j}$. Greek letters indicate a summation.

3. Mr. G. Y. Rainich: On the electromagnetic field in general relativity theory.

If at each point two perpendicular planes are given, we say we have a skeleton. A skeleton defines at each point two vectors $p$ and $q$. The principal planes of the tensors of an anti-symmetrical field constitute a skeleton; in order that an anti-symmetrical field satisfy the Maxwell equations it is necessary and sufficient that the vectors $p$ and $q$ be gradients. If we have a Maxwellian field a necessary and sufficient condition that the planes of its skeleton may be considered as tangent planes to two families of surfaces is that the vector $q$ be zero. The known energy relation which connects the contracted curvature tensor $F_{i j}$ with the electromagnetic tensor imposes on $F_{i j}$ the condition that the secular equation formed with the $F_{i j}$ have two positive and two negative roots all of the same absolute value. Geometrically it means that $F_{i j}$ defines a skeleton. The vector $p$ of this skeleton is a gradient as a consequence of the general properties of curved space, but the condition that $q$ be a gradient seems to impose a new condition on the space.

4. Professor R. E. Gilman: The remainder in Charlier's integral formulas.

The formula

$$
\begin{aligned}
& \int_{a}^{b} \lambda(x) d x=\omega \sum_{r=1}^{s-1} \lambda(a+r \omega)+\frac{1}{2} \omega[\lambda(b)-\lambda(a)] \\
&-\frac{\omega}{12}[\Delta \lambda(b)-\Delta \lambda(a)]+ \frac{\omega}{24}\left[\Delta^{2} \lambda(b)-\Delta^{2} \lambda(a)\right]+\cdots \\
& \cdots+C_{n} \omega\left[\Delta^{n} \lambda(b)-\Delta^{n} \lambda(a)\right]+R^{n}
\end{aligned}
$$

is typical of those considered. In this formula, $\lambda(x)$ is a single-valued function with a continuous $n$th derivative in the interval $a \leqq x \leqq b+n \omega, s$ is an integer, $\omega=(b-a) / s$, and $\Delta \lambda(a)$ has the customary meaning defined by the relations $\Delta \lambda(\alpha)=\lambda(a+\omega)-\lambda(a)$ and $\Delta^{k+1} \lambda(\alpha)=\Delta\left(\Delta^{k} \lambda(a)\right)$. An investigation of the remainder $R_{n}$ is carried out from two different points of view. The principal result of the paper is that 


$$
R_{n}=\omega^{n+1}(b-a) \lambda^{(n+1)}(\xi) \int_{0}^{1} \frac{t(t-1) \ldots(t-n)}{(n+1) !} d t,
$$

5. Professor Einar Hille: An existence theorem.

The integral equation of the Volterra type

$$
w(z)=w_{0}(z)+\int_{z}^{\infty} \sin (t-z) \Phi(t) w(t) d t
$$

is studied, in this paper, for complex values of $z$. The functions $w_{0}(z)$ and $\boldsymbol{\Phi}(z)$ are supposed to be single-valued and analytic in the strip $A<x, B_{1}<y<B_{2}(z=x+i y)$ and to be bounded in absolute value; $\Phi(z)$ in addition satisfies $|\boldsymbol{\Phi}(z)|<M /|z|^{1+\nu}, \nu>0$. The path of integration is a straight line parallel to the real axis. The existence is shown of a unique solution which is single-valued and analytic in the strip. The continuation of the solution outside of the strip in a sector is studied in the case in which $w_{0}(z)=c_{1} e^{i z}+c_{2} e^{-i z}$.

6. Professor E. W. Brown: A proof of the instability of the motions of asteroids whose periods are half that of Jupiter.

This paper consists mainly of an announcement of a proof of the instability of the motions of asteroids which have half the period of Jupiter. The well known gap in the distribution corresponding to this period has long been a puzzle. It appears that the instability is mainly one which causes eccentricities to increase so much that the configurations cannot be maintained.

7. Dr. C. A. Garabedian: Thick rectangular plates.

The author has recently presented a method of series in elasticity* in connection with its applications to Circular plates of constant or variable thicknesst and to Rods of constant or variable cross-section. . $^{\dagger}$ When the method is applied to rectangular plates the differential equations are partial instead of total, and the difficulties of integration are such that it is natural in a first presentation to avoid the complicated case of variable thickness. In the present note

- This Bulletin, April and May, 1923; Coмptes Rendus, vol. 177 (1923), p. 942.

$\dagger$ Transactions of this Society, July, 1923.

f This paper will appear in an early number of the AMERICAN Journal. 
the method of attack is illustrated by a computation of the exact solution for a rectangular plate of constant thickness, supported at the edges and under uniform pressure. The results will be found in the CoMPTES RENDUS in a note entitled Plaques rectangulaires épaisses. The author will show later how the method of series applied twice (secondly to the auxiliary partial differential equations which present themselves in the plate problem) leads to total differential equations and furnishes new solutions for beams of rectangular section.

8. Dr. F. H. Murray: Generalization of certain theorems of Bohl.

The object of this paper is the generalization of certain existence theorems obtained by $\mathrm{P}$. Bohl, concerning trajectories which remain in the neighborhood of a point of equilibrium for a one-sided variation of the time, or for all values of the time. When the equations in generalized coordinates of a dynamical problem are satisfied by a set of constant values of the variables, the theorems of Bohl cannot always be applied; in certain cases, however, similar theorems can be obtained by similar methods, and in this article a number of these are derived. They include the corresponding theorems of Bohl as special cases. (See P. Bohl, Über die Bewegung eines mechanischen Systems in der Nühe einer Gleichgewichtslage, CRELLE's JOURNAL, vol.127, pp. 179-276.)

9. Mr. A. D. Michal: Functionals of closed plane curves, invariant under one-parameter groups of transformations of the plane, and generalizations.

In a previous communication presented to the Society (Annual Meeting, 1923) the author commenced the study of functionals of closed plane curves invariant under a given arbitrary continuous one-parameter group of transformations of the plane. In the present paper the writer continues his discussion of such invariant functionals of closed plane curves. Furthermore the theory is extended to the case of functionals of closed plane curves depending in particular on a point on the curve. Generalizations of this invariant theory are effected in the case of functionals of closed $n-1$ spreads in $n$ dimensions. 
10. Mr. M. H. Stone: An unusual type of expansion problem.

In the present paper, the boundary value and expansion problems arising from the differential systems $u^{\prime}+\lambda p u=0$, $u(0)-u(1)=0,0 \leqq x \leqq 1$, and $u^{\prime}+\lambda p u=0, u(0)+u(1)=0$, $0 \leqq x \leqq 1$, are discussed. The function $p(x)$ is bounded, and is so restricted that the Lebesgue integral $P(x)=\int_{0}^{x} p d x$ has only a finite number of oscillations. The special case when $p$ is unity is taken up first. The general case is then considered. When $P$ is not monotone, the formal expansions of an arbitrary function represent, not the function itself, but a related function which may, in special cases, coincide with it. Infinitely many functions have the same formal expansions in terms of solutions of the two differential systems.

11. Mr. M. H. Stone: On the order of an analytic function at a singular point.

Hadamard in his Thèse defined the order of an analytic function at a singular point (JoURNAL DE MATHÉMATIQUES, ser. 4, vol. 8 (1892), pp. 101-186, part III). He did not prove the existence of the order. Later writers on the subject have neglected to give proofs, or have given incorrect proofs. In the present paper a demonstration of the existence of the order is given.

12. Professor J. R. Kline: Theorems concerning the division of a plane by continua.

Suppose the bounded decomposable continuum $M$, lying in the plane $S$, is such that if $g$ is any closed connected subset of $M$, then $M-g$ is connected. Under these conditions, the author proves that $M$ must divide the plane. He further proves that unless $M$ is a simple closed curve it cannot be a continuous curve.

13. Professor L. P. Eisenhart: Geometries of paths for which the equations of the paths admit a quadratic first integral.

A geometry of paths for a general space of $n$ dimensions is based upon the conception that the paths are fundamental 
entities. They are the integral curves of a system of differential equations of the form

$$
\frac{d^{2} x^{i}}{d s^{2}}+\Gamma_{\alpha \beta}^{i} \frac{d x^{\alpha}}{d s} \frac{d x^{\beta}}{d s}=0
$$

where $s$ is a special parameter for each path and the $\boldsymbol{\Gamma}_{\boldsymbol{\alpha} \beta}^{i}\left(=\boldsymbol{\Gamma}_{\beta \alpha}^{i}\right)$ are functions of the $x$ 's. The present paper determines the functions $\boldsymbol{\Gamma}_{\alpha \beta}^{i}$ in order that the above equations may admit a given quadratic first integral $g_{i j}\left(d x^{i} / d s\right)\left(d x^{j} / d s\right)$ = const. Veblen and Thomas have solved the converse problem: Given the functions $\Gamma$, to determine whether the equations admit a quadratic first integral.

14. Mr. W. E. Van de Walle: On the complete independence of the postulates for betweenness.

Of the eleven sets of postulates for betweenness published by Huntington and Kline in 1917 (Transactions OF THIS SocIETY, vol. 18, pp. 301-325), it is shown that sets 1-10 are completely independent, while set 11 is not. In the case of sets $1,2,3,5,6,7$, the necessary examples are given in terms of a class containing only four elements. In the case of sets $4,8,9,10,11$, some of the examples require the use of a class containing five elements. The failure of set 11 to be completely independent is due to the nonexistence of an example having the following "record": $A+, B+, C+, 3-, 4-, 8+$.

15. Professor J. F. Ritt: Equivalent rational substitutions.

This paper presents several results relative to systems of three rational functions $\varphi(z), \alpha(z), \beta(z)$, for which $\varphi[\alpha(z)]$ $=\varphi[\beta(z)]$.

16. Professor J. F. Ritt: New proofs of two well known theorems on quadratic forms.

Let $N$ represent a prime positive integer. It is well known that if $N \equiv 1, \bmod 4$, it has the form $\alpha^{2}+\beta^{2}$, where $\alpha$ and $\boldsymbol{\beta}$ are integers, and that if $N \equiv 1, \bmod 6$, it is of the form $\alpha^{2}+\alpha \beta+\beta^{2}$. The present note proves these results by an analytic method, which involves the construction of a Riemann surface, and its uniformization with elliptic functions. It is not believed that such a method has been used before in the theory of numbers. 
17. Professor R. L. Moore: A characterization of a bounded continuum which forms the common boundary of two domains in the plane.

It is shown that if, in a plane $S, M$ is a bounded continuum which has more than one prime part and no one of its prime parts separates $S$ then in order that $S-M$ should be the sum of two mutually exclusive domains such that every point of $M$ is a limit point of both of them it is necessary and sufficient that $M$ should contain no subcontinuum whose omission disconnects $M$.

18. Professor R. L. Moore: On the relation of a bounded continuum to its complement in the plane.

In a plane $\bar{S}$ let $\bar{M}$ denote a bounded continuum no one of whose prime parts separates $\bar{S}$. Let the term element denote any prime part of $\bar{M}$ or any point which does not belong to $\bar{M}$. Let $S$ denote the set of all elements and let $N$ denote the set of all prime parts of $\bar{M}$. An element $p$ is said to be a limit element of a set of elements $K$ if for every positive $\varepsilon$ there exists an element belonging to $K$ but distinct from $p$ and containing a point at a distance less than $\varepsilon$ from some point of $p$. It is shown that every "simple closed curve" of elements separates $S$ into two "domains," of which a particular one is called its interior. It is shown that if the interior of every simple closed curve of elements is called a region then all the axioms of the author's Foundations of plane analysis situs* are satisfied. It followst that between the elements of $S$ and the points of $\bar{S}$ there exists a one to one correspondence which preserves limits. The image $(\overline{N)}$ of $N$ under this correspondence is a continuous curve. Thus, from the stand-point of analysis situs, not only is $N$ a continuous curve (of elements) but its relation to the remainder of the plane is that of a continuous curve to its complement.

19. Professor J. W. Alexander: Note on Browwer's fixed point theorem.

Consider a continuous transformation $T_{1}$ of an $n$-sphere into itself such that no point is carried into its opposite.

* Transactions of this Society, vol. 17 (1916), pp. 131-164.

$\dagger$ Ibid., vol. 20 (1919), pp. 169-178. 
Each point $P$ may then be joined to its image $P^{\prime}$ by an arc of a great circle shorter than half a great circle; consequently, the transformation may be generated by a (singular) deformation and must be of index unity. Now, consider a continuous transformation $T_{2}$ with no fixed point. If $T_{2}$ be followed by a reflection, we obtain a transformation of the type $T_{1}$ and index one. The reflection is of index \pm 1 depending on whether the sphere is a manifold of an odd or even number of dimensions. Therefore, the index of $T_{2}$ must be \pm 1 depending on the dimensionality of the sphere.

20. Mr. G. Y. Rainich: On the dependence of the curvature upon the electromagnetic field.

Einstein introduces in his paper Bietet die Feldtheorie Möglichkeiten für die Lösung des Quantumproblems? (BERLIN SitzungsBerichte, December 13,1923 ) a certain expression 'I $\boldsymbol{S}_{i k, l m}$ which has the algebraic properties of the Riemann tensor and reduces to the energy tensor after contraction. If we designate by $\Omega_{i k, l m}$ the tensor which depends in the same way on the dual (magnetoelectric) tensor as $\Psi_{i k, l m}$ depends on the electromagnetic tensor, we have in $\Omega_{i k, l m}$ another expression with the same properties. We can form an infinity of linear combinations of these two tensors having the same properties. The expression proposed by the author in the December number of the ProceEdINGS of the National ACADEmy is a particular case of this, being equal to half of the difference of these two tensors.

R. G. D. Richardson, Secretary. 